\title{
Dehydroepiandrosterone 3-Glucuronide
}

National Cancer Institute

\section{Source}

National Cancer Institute. Dehydroepiandrosterone 3-Glucuronide. NCI Thesaurus. Code C120009.

A glucuronosyltransferase-catalyzed, liver metabolite of the steroid dehydroepiandrosterone (DHEA), which is an intermediate in the conversion of cholesterol to androgens and estrogens. 